\title{
The commercial tipping point
}

\author{
RNA-based therapeutics are poised to become successful commercial products, but wide adoption across the \\ biopharmaceutical industry will likely take a few more years.
}

\begin{abstract}
This is the decade of RNA-based therapy. At least, that is what was promised. In the past seven years, we have witnessed the approvals of three antisense oligonucleotides; two just in the past six months for Duchenne muscular dystrophy (Exondys 51) and spinal muscular atrophy (Spinraza). Next year, we expect the registration of the first short interfering RNA (siRNA) therapy (patisiran). Although there is gathering momentum, commercial success is by no means guaranteed.
\end{abstract}

This focus issue - together with a set of articles in Nature Reviews Drug Discovery-provides an overview of numerous RNA-based therapeutic modalities. As many of our authors profess, these molecules offer great promise in combatting human diseases previously intractable to therapy.

But commercial uptake is another matter. Typically, industry adoption of any new drug type is thought to follow the Gartner hype cycle; discovery is followed by a "peak of inflated expectations," a "trough of disillusionment," a subsequent "slope of enlightenment" and ultimately a "plateau of productivity."

To some extent, antisense and perhaps even siRNA have already negotiated their hype and disillusion phases. The antisense field witnessed the commercial failure of Vitravene and Kynamro and late-stage clinical failures of Genasense and Affinitak. The emergence in 2000 of Nobelprize-winning RNA interference (RNAi) technology also likely impeded antisense by deflecting investment away to newer, shinier siRNA companies. In their turn, RNAi drugs have had their fair share of letdowns: early clinical candidates foundered in wet age-related macular degeneration after concerns arose over innate immunostimulatory artifacts and delivery challenges. Exit big pharma pursued by a bear market!

With irrational exuberance now behind us, optimized chemistries and delivery vehicles are now laying out the path ahead. But where are RNA-based therapies on their ascent to commercial enlightenment?

One way to assess their progress is via a comparison to monoclonal antibodies (mAbs), which came of age in 1997 with the launch of Biogen's Rituxan (rituximab), a chimeric anti-CD20 mAb. By 2001, Rituxan had become the first billion-dollar blockbuster antibody, heralding the arrival of a new wave of products; 19 therapeutic mAbs were approved over the next five years, including Humira (adalimumab), which became the world's top-selling drug in 2012.

RNA-based drugmakers should be reassured that Rituxan's commercial success arrived over 20 years after the initial discovery of hybridomas by Kohler and Milstein. Establishing the commercial viability of a new therapeutic modality takes a long time. It also requires building confidence and awareness among clinicians across the globe beyond the select group involved in trials. In this respect, it is encouraging that the antisense field now has comparable numbers of companies with active development programs as the mAb field had 20 years ago. In 1997, 20 companies (including eight multinationals) were testing $\mathrm{mAbs}$ in $\sim 100$ clinical trials. Today, 18 companies (five of them big pharma) are developing antisense molecules in $~ 130$ clinical trials; 16 companies (including multinationals Sanofi, Pfizer and Bristol-Myers Squibb) are developing RNAi drugs in 28 clinical programs; and seven drug developers (including Baxter and Pfizer) are currently sponsoring ten clinical trials of aptamer drugs.

The above analysis suggests commercial activity in antisense today is comparable to that of the mAb field in 1997, just before it took off. But there are important differences.

One is intellectual property protection. For mAbs, the complexity of manufacture created an initial barrier to innovators but also erected a barrier to second movers. Subsequently, biosimilar legislation demanding comparable clinical performance rather than chemical equivalence (as for small-molecule drugs) created a further barrier to follow-on competitors. At the same time, the sluggishness with which that legislation was introduced gave $\mathrm{mAb}$ innovators market monopolies that extended well beyond patent protection. With antisense and siRNA, there is less patent protection from generic competition than with mAbs. On the other hand, constant innovation in oligo chemistry opens new patent vistas and rapid oligo design, discovery, preclinical validation and manufacture means less patent protection time is lost than is typical for small-molecule development.

Another fundamental difference lies in the contrasting therapeutic mechanisms of mAbs and RNA-based therapies. Antibodies act on extracellular targets and provide specificity of action through large surface area binding. The specificity of RNA-based therapies is conferred by Watson-Crick base pairing, but getting to the intracellular target and acting there is a much more complicated business. It involves the cellular uptake machinery, trafficking pathways and enzymes, such as RNase $\mathrm{H}$ and RISC. As human biology has limitless ways of providing surprises to drug developers, the dependence of RNA-based treatments on partially understood intracellular mechanisms thus poses a higher risk of failure.

What's more, the need to avoid the reticulo-endothelial system, cross cell membranes and find a way to exit endosomes constrains current RNA-based therapies to addressing disorders of the liver, eye, joints and central nervous system (CNS). Of course, this still leaves plenty of commercially attractive targets - and CNS disorders remain a large, and largely virgin, territory.

None of the above factors is insurmountable. But they should give pause to those suggesting that a raft of RNA-based therapies are about to take the world by storm. Spinraza promises to be a commercial success. But even if it is, for the wider industry to take note, that success will have to be repeated many times over.

RNA-based therapies are powerful adjuncts to the drug developer's existing toolbox of small molecules, recombinant proteins and mAbs. But commercial success is constructed one indication and one investment program at a time. While this decade may not witness a landslide of RNA-based therapies, watch this space in the 2020s! 